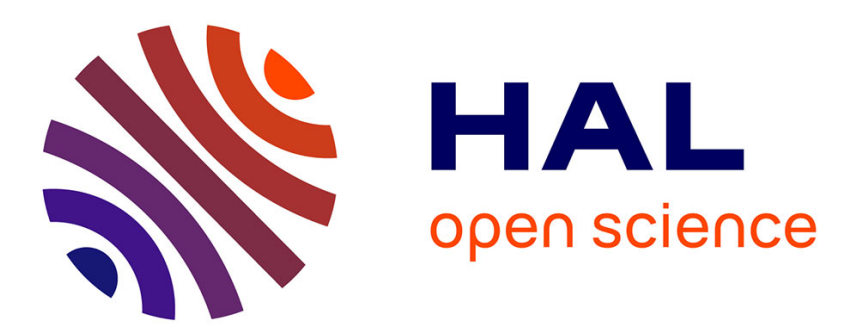

\title{
Cost, power consumption and performance evaluation of metro networks
}

Andrea Bianco, Thomas Bonald, Davide Cuda, Raluca-Maria Indre

\section{To cite this version:}

Andrea Bianco, Thomas Bonald, Davide Cuda, Raluca-Maria Indre. Cost, power consumption and performance evaluation of metro networks. Journal of Optical Communications and Networking, 2013, 5 (1), pp.81-91. hal-00784181

\section{HAL Id: hal-00784181 https://hal-imt.archives-ouvertes.fr/hal-00784181}

Submitted on 9 Mar 2016

HAL is a multi-disciplinary open access archive for the deposit and dissemination of scientific research documents, whether they are published or not. The documents may come from teaching and research institutions in France or abroad, or from public or private research centers.
L'archive ouverte pluridisciplinaire HAL, est destinée au dépôt et à la diffusion de documents scientifiques de niveau recherche, publiés ou non, émanant des établissements d'enseignement et de recherche français ou étrangers, des laboratoires publics ou privés. 


\title{
Cost, power consumption and performance evaluation of metro networks
}

\author{
Andrea Bianco ${ }^{1}$, Thomas Bonald ${ }^{2}$, Davide $\mathrm{Cuda}^{2}$, and Raluca-Maria Indre ${ }^{3}$ \\ ${ }^{1}$ Politecnico di Torino, Italy, andrea.bianco@polito.it \\ ${ }^{2}$ Telecom ParisTech, France, \{thomas.bonald, davide.cuda\}@ telecom-paristech.fr \\ ${ }^{3}$ Orange Labs, France, ralucamaria.indre@ orange.com
}

\begin{abstract}
We provide models for evaluating the performance, cost and power consumption of different architectures suitable for a metropolitan area network (MAN). We then apply these models to compare today's SONET/SDH metro rings with different alternatives envisaged for next-generation MAN: an Ethernetcarrier grade ring, an optical hub-based architecture and an optical time-slotted WDM ring. Our results indicate that the optical architectures are likely to decrease power consumption by up to $75 \%$, when compared to present day MANs. Moreover, by allowing the capacity of each wavelength to be dynamically shared among all nodes, a transparent slotted WDM yields throughput performance which is practically equivalent to that of today's electronic architectures, for equal capacity.
\end{abstract}

\section{INTRODUCTION}

The growing ubiquity of broadband access solutions such as Fiber To The Home (FTTH) is currently putting a strain on metropolitan area network (MAN) bandwidth. Most of today's metropolitan area networks are based on circuitswitched Synchronous Optical Network/Synchronous Digital Hierarchy (SONET/SDH) architectures. Initially designed to transport constant-rate voice traffic, SONET/SDH networks are unable to cope with today's constantly increasing bursty data traffic demands. Thus, Internet Service Providers (ISP) deploy Ethernet-based metropolitan area networks which are arguably more flexible, scalable and cost-effective than legacy SONET/SDH architectures [1], [2], [3]. Despite these advantages, Ethernet-based MAN still represents an opaque solution because optical signals must be converted to the electronic domain at every intermediate network node. Optical-electronicoptical conversion represents the largest cost when operating optical fiber networks [4]; thus, reducing or eliminating $\mathrm{O} / \mathrm{E} / \mathrm{O}$ conversion of in-transit traffic is a key design objective for MANs.

In today's metro networks most of the traffic carried by the network is destined to the Internet. In this context, $\mathrm{O} / \mathrm{E} / \mathrm{O}$ conversion at intermediate nodes can be eliminated simply using a dedicated wavelength channel to connect each network node to a hub node which provides access to the Internet. We refer to this architecture as hub-based architecture. Although this simple solution can readily be implemented using existing optical devices, it is hampered by severe scalability issues. First, the technology most likely to be deployed in the metro area is coarse WDM (CWDM) which only supports up to 16 wavelengths. Thus, the number of nodes can not exceed the number of available wavelengths, unless exploiting spatial diversity with parallel fibers. Second, the whole inbound traffic, i.e., traffic not destined to the Internet, undergoes unnecessary $\mathrm{O} / \mathrm{E} / \mathrm{O}$ conversion and processing at the hub node.

Scalability can be improved by allowing the capacity of each wavelength channel to be shared by multiple access nodes. Sharing can be achieved in the time domain by dividing the wavelength capacity into fixed-size time-slots. Time-slotted WDM architectures have been demonstrated as a viable approach to provide all-to-all connectivity in optical fiber rings [5], [6], [7], [8], [9], [10], [11], [12]. Slots propagate on the ring and each node can add/drop data to/from in-transit slots by means of one optical transmitter and receiver pair which we also refer to as transceiver. Tunability is required at least at the transmitter or receiver side to enable single-hop, alloptical, connections between nodes. Since the architectures in [5], [6], [7] require several transceivers per node, and [8] does not support spatial reuse, we consider the architecture proposed in the ECOFRAME project [13]. This architecture, named slotted WDM in the following, requires a single tunable transmitter and a fixed receiver per node and provides great flexibility, being able to dynamically adapt the wavelength load to changing traffic demands.

Since both the hub-based and slotted WDM solutions allow in-transit traffic to transparently bypass intermediate nodes, we refer to them as transparent architectures. In this paper, we provide models to compare performance, cost and power consumption of the above described architectures. We apply these models to a realistic case study and compare the two opaque solutions (SONET/SDH and Ethernet Carrier Grade) to the two transparent solutions (hub-based and time-slotted WDM) in the context of ring MANs. Specifically, we consider a MAN in which network nodes are interconnected by means of two counter-rotating fiber rings. One of these rings is used exclusively for protection purposes. In our study, we only concentrate on the operation of a single ring; the one used for transporting data.

We show that by allowing in-transit traffic to optically bypass intermediate nodes, the two transparent solutions require a smaller number of electronic components, thus reducing network cost and power consumption. Furthermore, we analytically prove that a slotted WDM solution is able to efficiently utilize network resources attaining a traffic capacity which is 
comparable to that of today's electronic networks.

The remainder of this paper is organized as follows. The next section describes the considered network architectures. In Sec.III we present a model for cost and power consumption evaluation. The model for performance evaluation is then presented in Sec.IV, whereas in Sec.V we present a case study highlighting the expected performance, cost and power consumption of each architecture. Sec.VI concludes the paper.

\section{NETWORK ARCHITECTURE}

We describe the four considered metro network architectures, composed by $N$ access nodes, which connect the access networks to the metro network, and a hub node which connects the metro network to the Internet. The $N$ access nodes and the hub node are interconnected through optical fiber links forming a uni-directional ring topology. On each link $W$ wavelengths are available, each operating at $R$ bit/s which represents the bit rate of the transceivers (i.e., a transmitterreceiver pair). The number of wavelengths $W$ and the bit rate $R$ are different for each architecture, as shortly explained, but the overall network capacity is the same to ensure a fair comparison.

\section{A. Opaque architectures}

Two different opaque technologies are considered: SONET/SDH and Carrier Grade Ethernet. As shown in Fig.1, in an opaque architecture each wavelength channel is terminated at each node. Both the access nodes and the hub node need to be equipped with $W$ fixed transceivers operating at $R \mathrm{Gbit} / \mathrm{s}$ (FTx-FRx). Network nodes are interconnected by point-to-point optical links, meaning that the transmitted signal undergoes $\mathrm{O} / \mathrm{E} / \mathrm{O}$ conversion at every network node.

SONET/SDH: The majority of currently deployed metropolitan area networks rely on the SONET/SDH architecture, that owes its popularity to its well-established standards, survivability, reliability, and performance monitoring features [14]. SONET/SDH is a circuit-based technology that supports bit rates of up to $40 \mathrm{Gbit} / \mathrm{s}$. It is based on a digital time division multiplexing (TDM) signal hierarchy where periodic time-frames of $125 \mu$ s carry payload traffic of various rates.

In Sec.III-A, the architecture of SONET/SDH nodes is described in more detail to evaluate the cost and power consumption of a SONET/SDH ring network.

Carrier Grade Ethernet : Since circuit-based SONET/ SDH networks can no longer efficiently sustain today's rapidly growing bursty data traffic, Internet service providers are showing increasing interest in Carrier Grade Ethernet as a solution to build more scalable and dynamic metropolitan area networks. Ethernet-over-fiber architectures have recently been the subject of many case studies [1], [3], [15]. For instance, a Metro Ethernet Forum study [1] shows that due to its operational simplicity, data transport efficiency and scalability, Ethernet-over-fiber may yield $50 \%$ operational expense (OPEX) and 40\% capital expense (CAPEX) savings as compared to legacy SONET/SDH solutions.

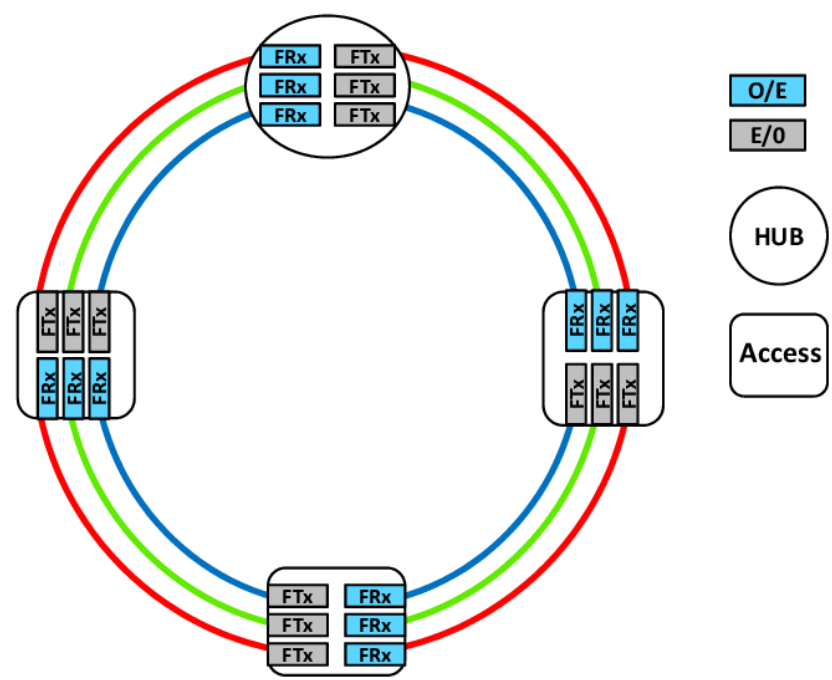

Fig. 1. An opaque architecture with one hub, $N=3$ access nodes and $W=3$ wavelength channels.

The network nodes are Carrier Grade Ethernet switches able to process incoming Ethernet frames and switch them towards their corresponding outputs. As in the case of the SONET/SDH network, all Ethernet network nodes perform $\mathrm{O} / \mathrm{E} / \mathrm{O}$ conversion of every wavelength and are thus equipped with $W$ transceivers.

\section{B. Transparent architectures}

Costly O/E/O conversion can be avoided by simply allowing in-transit traffic to optically bypass intermediate nodes. Several solutions are available to introduce transparency in an optical ring. We present here two possible approaches.

Hub-based optical ring: The traffic inserted by an access node is typically either local, i.e., directed to one of the other access nodes on the ring, or transit traffic, i.e., directed to the Internet. In today's metropolitan area networks, most of the traffic is transit traffic, i.e., being destined and/or originating from the hub node. Thus, a simple manner to introduce transparency is allowing every access node to have a direct optical connection to the hub node. We refer to this optical connection as a lightpath, i.e., a specific wavelength channel which spans one or several fiber links. Data transmitted by access nodes is converted to the optical domain at the source node and it is only converted back to the electric domain when it reaches the hub node, with no conversion at intermediate nodes. The same wavelength channel, but on different fiber links, can be used to transport downstream traffic, i.e., traffic coming from the Internet towards the access nodes (see Fig.2). Thus, the number of lightpaths on each fiber link is equal to the number of access nodes, i.e., $W=N$. Since lightpaths are not shared, they can operate at a lower bit rate than the wavelengths used in the other transparent architecture, as discussed later. 
Each access node is a static Optical Add Drop Multiplexer (OADM), able to send and receive traffic on a pre-defined wavelength channel. Thus, the OADM must contain an optical multiplexer/demultiplexer (MUX/DMUX). An access node only drops (i.e., extracts) its corresponding wavelength from the aggregate WDM signal. All other wavelengths optically bypass the node. Thus, each node is equipped with a single transceiver operating at $R \mathrm{Gbit} / \mathrm{s}$ (FTx-FRx). The queuing and aggregation requirements of access nodes can be satisfied in the electronic domain by a simple Ethernet switch that aggregates incoming traffic, without requiring any complex processing.

In the hub-based architecture, access nodes send both local and transit traffic to the hub node. Thus, the hub is a highcapacity switch that must terminate and switch the entire network traffic aggregate. Unlike in previous architectures, the size and complexity of the hub node grows proportionally with the number of access nodes. More precisely, the hub node must be equipped with $N$ fixed transceivers (FTx-FRx) of $R$ Gbit/s such that it can manage $N$ lightpaths, one per access node. Obviously, this architecture is suited to the current network traffic scenario, in which most of the traffic is either destined or coming from the Internet.

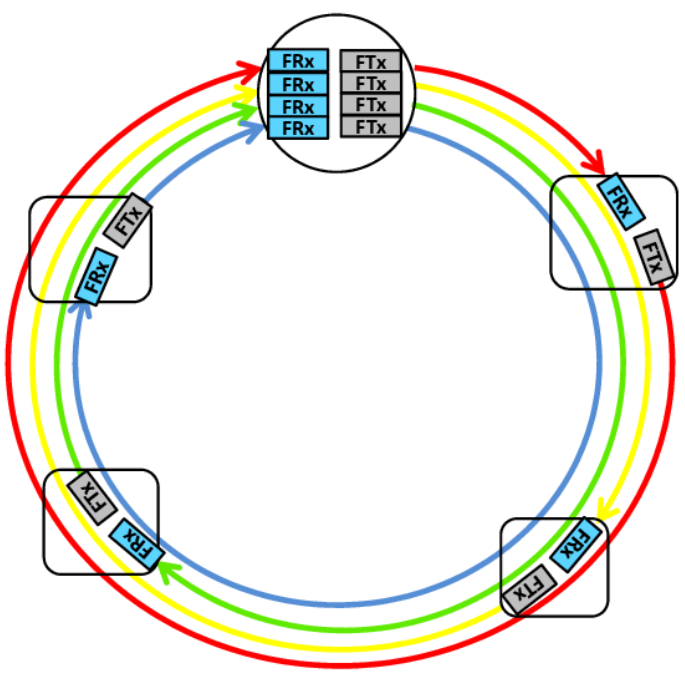

Fig. 2. A hub-based transparent optical ring with $N=4$ access nodes and $W=N=4$ wavelength channels.

Time-slotted WDM ring: The hub-based architecture eliminates the need for $\mathrm{O} / \mathrm{E} / \mathrm{O}$ conversion at intermediate nodes, but it is not scalable and not easily adaptable to dynamic traffic scenarios. These drawbacks can be overcome by allowing the capacity of each channel to be shared between multiple source-destination nodes. Sharing can be achieved e.g., in time domain, by dividing the wavelength capacity into fixedsize time-slots propagating on the ring. Each network node inserts/extracts traffic to/from specific time-slots based on their availability.

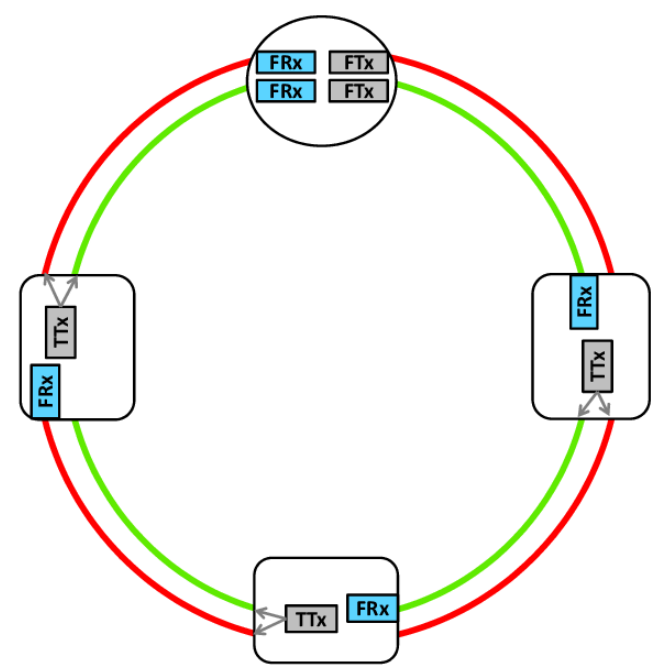

Fig. 3. A time-slotted WDM ring with $N=3$ access nodes and $W=2$ wavelength channels.

We focus on the particular network architecture proposed within the ECOFRAME project [13]. In this architecture, each node is a Optical Packet Add Drop Multiplexer (OPADM) able to insert (add) and to extract (drop) traffic from timeslots on one or several wavelength channels. The OPADMs are connected by WDM fiber links that carry $W$ wavelengths. Every access node is able to transmit on all $W$ wavelength channels, via a tunable transmitter (TTx), but it is able to receive on a single pre-defined channel thanks to a fixed receiver (FRx). The hub node is able to simultaneously receive and transmit data on all $W$ wavelengths being equipped with $W$ fixed transceivers (FTx-FRx). If the number of available wavelengths $W$ is smaller than the number of access nodes $N$, then each wavelength must be shared in reception by several nodes. Ideally, wavelengths should be allocated such that the traffic is equally balanced over all wavelengths. In the following we consider that each wavelength channel is shared in reception by $K=N / W$ access nodes such that all wavelengths are equally loaded for uniform traffic. Since wavelengths represent a shared medium, an access protocol must be implemented to avoid collisions. We consider here a greedy access scheme in which nodes seize every opportunity to use an empty slot in order to send waiting data packets. This greedy scheme may lead to starvation, as shown in [12], for instance. However, as we shall see in Sec.V, the link capacity is several orders of magnitude higher than the typical flow peak rate which makes starvation scenarios very unlikely .

\section{COST AND POWER CONSUMPTION}

We now focus on the evaluation of the cost and the power consumption of the four architectures presented in the previous section. We propose a discrete component approach to evaluate the cost of the considered architectures, both in terms of the Capital Expenditures (CAPEX) and in terms of consumed power part of Operational Expenditures (OPEX). The cost 


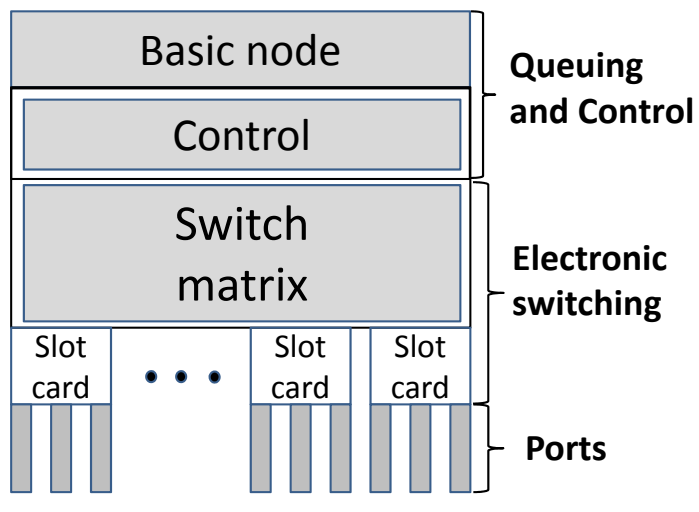

Fig. 4. Building blocks of an opaque network node.

model utilized in this study is inspired by the CAPEX model used in the NOBEL research project [16], [17].

\section{A. Opaque architectures}

The cost of an opaque network node can be assessed by subdividing this node into its main building blocks: the base node and the equipment related to physical layer interfaces. The base node includes the chassis, the physical and mechanical assembly, the switching matrix, the cooling, the power supplies, as well as the control and the management software. Each base node provides a limited number of slots for physical-layer interfaces. Each slot must be equipped with a transponder slot card which provides the capability of connecting different types of transceivers. The transceivers considered here are $10 \mathrm{Gbit} / \mathrm{s}$ small form factor pluggables (XFP) [18] with a transmission range of $40 \mathrm{~km}$, a reasonable distance for a metro network. Fig.4 depicts the structure of an opaque node.

An access node has two types of physical-layer interfaces: interfaces that connect the access node with a downstream access network and interfaces which are used to connect the access node to the other metro nodes. The connection to the other metro nodes is provided by means of $W=2$ wavelengths operating at $R=10 \mathrm{Gbit} / \mathrm{s}$. The hub is also connected to two access nodes via $W=2$ wavelengths of $R=10 \mathrm{Gbit} / \mathrm{s}$. Furthermore, the hub connects the metro ring to the Internet. Its switching capacity is thus higher than that of the access nodes.

The total cost and power consumption of an opaque network node are obtained by simply summing up the cost and power consumption of the base node, slot cards and transceivers.

\section{B. Transparent architectures}

As for the opaque network nodes, we asses the cost and power consumption of OADMs and OPADMs by decomposing these nodes into their main building blocks. Fig.5 shows the structure of an OPADM [13] used in the time-slotted WDM architecture. It consists of two multiplexer/demultiplexers, a fixed Burst Mode Receiver (BMR), $W$ Semiconductor Optical Amplifier (SOA), a tunable transmitter (TTx) plus a fixed transceiver (FTx-FRx) for the control channel. The first
MUX/DMUX allows to separate the $W$ wavelength channels of the composite WDM signal. Each access node is able to receive on a specific wavelength channel $w$. This wavelength is locally dropped by means of a fixed receiver which operates in burst mode. The SOAs are used both as an amplifier and as an on-off gate. Specifically, if a node wants to insert data on a wavelength $w$ in time-slot $s$, then the SOA is used to erase the data contained by the time-slot $s$ on $w$ so as to allow the transmitter to insert data in this time-slot. If node $i$ does not transmit in slot $s$, the SOA is used to amplify the intransit signal. The SOA is controlled by an electronic control unit which receives control information about each slot via the control channel. The tunable transmitter is used to send data on one of the $W$ wavelength channels but it can only send data on a single wavelength in each time-slot. Finally, the second MUX/DMUX multiplexes the optical signal and sends it via the output fiber. We assume that a basic Ethernet aggregation switch provides the required queuing and aggregation capacity.

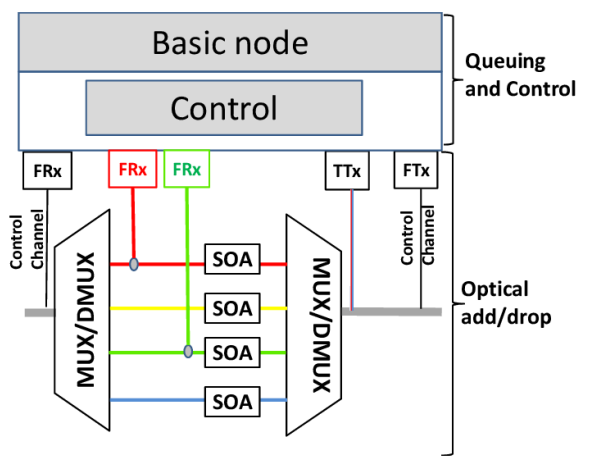

Fig. 5. The structure of an OPADM receiving on 2 distinct wavelength channels.

The cost of an OPADM can be evaluated by simply adding the cost of discrete components. Note that this approach does not consider on-chip integration and can thus be considered as a rough guide to assess a worst-case cost of the slotted WDM solution. The CAPEX and consumed power costs are estimated by adding up the cost of the following components: base node, multiplexer, splitters, SOA gates, tunable transmitter and burstmode receiver needed for the payload and a fixed transceiver required for the control channel. Note that the number of splitters and BMR increases linearly with the number of dropped wavelengths.

The architecture of the OADM node used in the hub-based architecture is much simpler, since it only requires a base node for queuing and aggregation plus two MUX/DMUX, one fixed transmitter and one continuous mode receiver. The cost and power consumption can once more be obtained by adding up the cost/power consumption of each individual component.

\section{Performance evaluation}

In this section we evaluate the performance of the considered network architectures in terms of their ability to transport 
data traffic. More precisely, we focus on two performance metrics that we name traffic capacity and maximum sustainable traffic to obtain a given target congestion rate.

\section{A. Traffic capacity}

We define the traffic capacity as the stability limit beyond which the size of the waiting queues would grow indefinitely. For a network to be stable, the offered traffic should not exceed the transmission and reception capabilities of each network node. We recall that the transmission and reception capabilities of an access node are different in each of the considered network architectures. Specifically, in the opaque architectures, each node is equipped with $W$ fixed transceivers, while the access nodes of the transparent architectures only require a single transceiver. The hub node is equipped with $W$ transceivers in all architectures.

We denote by $t_{i}, t_{i} \subset\{1, \ldots, W\}$, the set of transmitters at node $i, i=0,1, \ldots, N$ and by $r_{i}, r_{i} \subset\{1, \ldots, W\}$ the set of fixed receivers. Index $i=0$ corresponds to the hub node. Node $i$ can thus simultaneously transmit data on $\left|t_{i}\right|$ wavelengths and receive data on $\left|r_{i}\right|$ distinct wavelength channels. We further denote by $a_{i j}$ the offered traffic on source-destination pair $(i, j)$ and by $a_{i}=\sum_{j} a_{i j}$ the total traffic injected by node $i$. Finally, we refer to link $l$ as the link connecting node $l$ to node $l+1$, for all $l=0, \ldots, N-1$. We denote by $[i, j]$ the set of nodes between node $i$ and node $j$, that is the set $\{i, \ldots, j\}$ if $i \leq j$ and the set $\{i, \ldots, N\} \cup\{1, \ldots, j\}$ otherwise. To ensure stability, the traffic generated and received by each node $i$ must not exceed node's $i$ transmission and reception capacity:

$$
\forall i, \quad a_{i}<\left|t_{i}\right| R
$$

and

$$
\forall i, \quad \sum_{j} a_{i j}<\left|r_{i}\right| R
$$

where $R$ is the transceiver speed.

Another condition that must be verified for stability is that the total traffic going through each shared resource (i.e., network link or wavelength) is less than the resource capacity.

In the hub-based architecture, wavelength channels are dedicated to a specific source-destination pair such that conditions (1) and (2) are sufficient for stability. In particular, for each access station we verify (1) and (2) with $t_{i}=1$ for all $i \geq 1$ with $r_{i}=1$ for all $i \geq 1$. Recall that the hub station has as many transceivers as there are nodes such that the above conditions is verified for $t_{0}=N$ and $r_{0}=N$.

In the case of the opaque architectures, each network link is shared by all the source-destination pairs going through that link, yielding an additional stability condition:

$$
\forall l, \quad \sum_{(i, j): l \in[i, j]} a_{i j}<W R .
$$

The above condition must also be verified in the timeslotted WDM architecture. Additionally, since each individual wavelength channel is shared by specific source-destination pairs we also need to verify that on every wavelength $w$ the offered traffic is inferior to the wavelength capacity, yielding:

$$
\forall w, \forall l, \quad \sum_{(i, j): l \in[i, j], r_{j}=w} a_{i j}<R .
$$

The above described stability conditions define the traffic capacity of the considered optical ring networks. Note that in the case of time-slotted WDM rings with opportunistic access these conditions may not always be sufficient for stability. However, in the following we do not consider such specific traffic scenarios and thus the above conditions are sufficient for network stability.

\section{B. Congestion rate}

In the previous section we focused on the maximum traffic that a network can sustain based on stability conditions. We are now interested in computing the maximum sustainable traffic such that a certain performance level can be attained. The performance metric considered here is the congestion rate defined as the probability that the traffic offered to a specific shared resource exceeds the capacity of that resource. We focus on how network links are shared by flows belonging to different source-destination pairs.

We consider a flow-level model and we assume that flow arrivals follow a Poisson process of intensity $\lambda$ with an exponential duration of average $1 / \mu$. We denote by $\alpha=\lambda / \mu$ the traffic intensity in Erlang. We assume that all flows have the same peak rate $r$ and share a link of capacity $C$, where $C=m r$ is a multiple of the flow peak rate. The specific value of $C$ depends on the considered architecture. For instance, in the case of the opaque architectures, the shared resource is the network link and thus $C=W R$ as shown in (3). The traffic intensity in bit/s is given by $A=\alpha r$. The link load is simply the total offered traffic divided by the link capacity:

$$
\rho=\frac{A}{C}=\frac{\alpha}{m} .
$$

The congestion rate represents the probability that the aggregate rate of the flows that share the considered link is larger than the link capacity. Considering a link of capacity $C=m r$, the link is limiting whenever the number of transmitted flows is higher than $m$. As long as $x<m$, each flow can transmit at its peak rate. If $x$ exceeds $m$, then the link is shared fairly among the $x$ flows and each flow has a rate of $C / x$. Under the above assumptions of Poisson flow arrivals and exponential flow size, this model corresponds to an $M / M / m$ queue with processor sharing (PS) service discipline. Thus, the number of ongoing flows is given by:

$$
\pi(x)=\pi(0) \frac{\alpha^{x}}{x !}, \text { if } x \leq m
$$

and

$$
\pi(x)=\pi(m) \rho^{x-m} \text {, if } x>m .
$$

After normalization, we obtain: 


$$
\pi(0)=\frac{1}{1+\alpha+\frac{\alpha^{2}}{2}+\ldots+\frac{\alpha^{m}}{m !}+\frac{\alpha^{m}}{m !} \frac{\rho}{1-\rho}} .
$$

The congestion rate is the probability that the total rate of the flows exceeds the link capacity, namely:

$$
G=P(x r>C)=P(x>m)=\sum_{x>m} \pi(x) .
$$

The duration of each flow may depend on the network state. However, for fairly low congestion rates, when the percentage of lost packets is small and can thus be neglected, we can assume that the duration of each flow does not depend on the network state. The flow level model now corresponds to an $M / M / \infty$, i.e., a queue with an infinite number of servers. In this simpler case, the number of flows in the system is given by:

$$
\pi(x)=e^{-\alpha} \frac{\alpha^{x}}{x !}
$$

The congestion rate is still expressed as:

$$
G=\sum_{x>m} \pi(x)
$$

In the following, we use the $M / M / m$ to model network performance under relatively high congestion rates and the $M / M / \infty$ to model the performance at low congestion rates, say $1 \%$.

\section{CAse Study}

We now consider a specific case study to which we apply the previously described models. Our goal is to compare performance, cost and power consumption of the four considered network architectures. To ensure a fair comparison, we assume that all architectures have the same number of nodes and the same total network capacity. We first describe the considered network parameters. We then apply the performance and cost models presented in the previous two sections.

\section{A. Network parameters}

We consider ring networks of $N$ access nodes plus an extra hub node providing access to the Internet. We assume that, in the SONET/SDH, Ethernet and the time-slotted WDM architectures, access nodes are interconnected by $W_{1}$ wavelengths operating at $R_{1} \mathrm{Gbit} / \mathrm{s}$. Recall that nodes in the opaque architectures are equipped with $W_{1}$ transceivers while in the transparent architectures nodes are equipped with a single tunable transmitter and a fixed receiver. In the hub-based architecture the number of wavelengths is equal to the number of nodes, i.e., $W_{2}=N$ and each access node is connected to the hub node by a dedicated lightpath of capacity $R_{2} \mathrm{Gbit} / \mathrm{s}$. To ensure that all four architectures have the same aggregate network capacity, the following condition needs to be verified:

$$
W_{1} R_{1}=W_{2} R_{2}
$$

\section{B. Traffic scenarios}

We want to evaluate network performance under typical traffic conditions. To this end, we define the following three traffic scenarios depicted in Fig.6:

Local scenario: Only access nodes exchange traffic (i.e., no traffic is generated by or directed to the hub) and traffic is uniformly distributed over all stations, yielding:

$$
\forall i, j \neq 0, i \neq j, \quad a_{i j}=\frac{A}{N(N-1)} .
$$

Transit scenario: All traffic is transit traffic, either received from or directed to the Internet. Thus, a node $i \neq 0$ sends all traffic to the hub. Referring to "upstream" as the traffic directed to the hub and to "downstream" as the traffic originating from the hub, the traffic matrix is characterized by a single parameter $\beta$ representing the fraction of upstream traffic:

$$
\forall i \neq 0, \quad a_{0 i}=\frac{A(1-\beta)}{N}, \quad a_{i 0}=\frac{A \beta}{N} .
$$

Hybrid scenario: Any pair of nodes $(i, j)$ exchange symmetric traffic: $\lambda_{i j}=\lambda_{j i}$ for all $i, j, i \neq j$, which is representative of a peer-to-peer traffic scenario. The traffic matrix is characterized by a single parameter $\eta$ representing, for any node $i \neq 0$, the fraction of local traffic, i.e., the fraction of traffic destined to all nodes $j, j \neq 0$ :

$$
\forall i \neq 0, \quad a_{0 i}=a_{i 0}=\frac{A(1-\eta)}{2 N},
$$

and

$$
\forall i, j \neq 0, i \neq j, \quad a_{i j}=\frac{A \eta}{N(N-1)} .
$$

Unless otherwise specified, we take $\beta=\eta=20 \%$ for both the transit and the hybrid scenario.
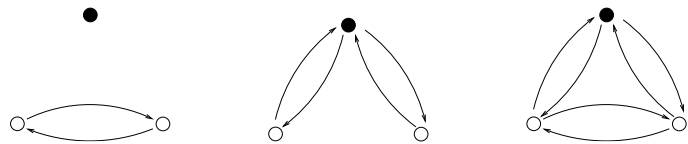

Fig. 6. Considered traffic scenarios (from right to left): local, transit and hybrid (hub station represented in black and access nodes in white)

\section{Performance evaluation}

The traffic capacity is defined by the stability conditions (1)(3). Since the two opaque architectures are equivalent in terms of network resource sharing, we do not distinguish between them in the analysis. However, the SONET/SDH architecture provides connectivity by means of circuit-switching and it is likely to be very sensitive to traffic burstiness, a factor which is not considered in the present model.

We first focus on the Local traffic scenario. In the opaque architectures, the most constraining condition is condition (3) which defines the maximum traffic which can be sustained by each network link. Since in the opaque architectures for local traffic all links are equally loaded, we write condition (3) for an opaque architecture on link 0, i.e., the link between the hub and access node 1 . We have: 


$$
\sum_{j=1}^{N-1} \sum_{i>j}^{N} a_{i j}<W_{1} R_{1} .
$$

The maximum traffic that can be injected by a node $i$ is:

$$
a_{i}=(N-1) a_{i j}=\frac{2 W_{1} R_{1}}{N} .
$$

This corresponds to a traffic capacity of $2 W_{1} R_{1}$. Note that the traffic capacity is twice the network capacity. This is due to the spatial reuse factor of 2, typical of ring architectures with destination stripping, i.e., destinations immediately release the slot on which they receive data.

In the case of the hub-based architecture, the traffic capacity of the network is limited by the reduced transmission and reception capacity of each node. Since access nodes are connected to the hub via lightpaths operating at $R_{2} \mathrm{Gbit} / \mathrm{s}$, the access node can insert at most $R_{2} \mathrm{Gbit} / \mathrm{s}$, yielding a total traffic capacity of $W_{2} R_{2}$ Gbit/s. Since the hub-based architecture does not allow spatial reuse, its traffic capacity represents only half of the traffic capacity of the opaque network.

Finally, we evaluate the traffic capacity of the slotted WDM architecture. Since the number of wavelengths $W_{1}$ is typically inferior to the number of nodes $N$, each wavelength is shared in reception by $K=N / W_{1}$ different nodes. Let us consider the simple case of a network in which $K$ is an integer. Assume that the received wavelengths are allocated in a cyclic manner from node 1 to node $N$. The most constraining stability condition is given by (4). We deduce the total traffic capacity:

$$
\frac{2 W_{1} R_{1}(N-1)}{N+W_{1}-2} \text {. }
$$

Note that for large values of $N$ and fixed $W_{1}$, the traffic capacity tends to $2 W_{1} R_{1}$ which represents the traffic capacity of the opaque architecture. Note also that for the same network capacity $W_{1} R_{1}$ and the same number of nodes $N$, the traffic capacity decreases as the number of wavelength channels increases: When $W_{1}=N$ (corresponding to $K=1$ ), there is a single node per wavelength and the traffic capacity takes its minimum value of $W_{1} R_{1}$ (no spatial reuse).

We now consider the Transit scenario. For all architectures, the most loaded network link is link 0. Therefore, we must verify that stability condition (3) holds for this link, namely:

$$
\sum_{i=1}^{N} a_{0 i}<W_{1} R_{1} .
$$

In view of (7) and (12), the maximum traffic capacity is given by:

$$
\frac{W_{1} R_{1}}{\beta} .
$$

The traffic capacity is the same for all the considered architectures, because they share the same stability condition.

The Hybrid scenario is simply a combination of the previous two traffic scenarios. Its traffic capacity can be computed in a similar way.

\begin{tabular}{|c|c|c|c|}
\hline & Local (Gbit/s) & Transit (Gbit/s) & Hybrid (Gbit/s) \\
\hline \hline Opaque & 40 & 25 & 40 \\
\hline Hub-based & 20 & 25 & 33 \\
\hline Slotted WDM & 38 & 25 & 40 \\
\hline
\end{tabular}

TABLE I

TRAFFIC CAPACITY OF EACH ARCHITECTURE UNDER THE LOCAL, TRANSIT AND HYBRID TRAFFIC SCENARIOS.

To illustrate the traffic capacity of the considered architectures under the above traffic scenarios, we consider a specific case study. We assume a network of $N=20$ access nodes and one hub station. In the opaque and time-slotted WDM architecture, these nodes are interconnected by $W_{1}=2$ wavelength channels operating at $R_{1}=10 \mathrm{Gbit} / \mathrm{s}$ each. In the hub-based architecture, every access node is connected to the hub station via a dedicated wavelength channel. There are thus $W_{2}=N=20$ wavelength channels and each of these channels operates at $R_{2}=1 \mathrm{Gbit} / \mathrm{s}$, such that (5) is verified. Tab.I gives the total traffic capacity for each of the considered architectures under the three traffic scenarios.

We first note that under the Local traffic scenario, the traffic capacity of the time-slotted WDM is slightly smaller than the capacity of opaque architectures. Indeed, since in the slotted WDM architecture each wavelength corresponds to a specific set of destinations, wavelengths are not equally loaded on each link. This constraint slightly impacts the traffic capacity which is bounded by the most loaded wavelength.

In the Transit traffic scenario in which most of the traffic is directed to the hub node, the hub-based architecture attains the same traffic capacity as the opaque and time-slotted WDM architectures. However, when the proportion of local traffic increases, the slotted WDM and the opaque architectures outperform the hub-based architecture. This is because the opaque and the slotted WDM architecture allow spatial reuse of the available bandwidth, thus increasing the traffic capacity. For instance, in the slotted WDM architecture the same timeslot can be used for transmission from the hub node to a node $i$ and from node $j$ to the hub, with $j>i$. This reuse is not possible in the hub-based architecture in which every wavelength is statically assigned to a pair of network nodes. Note that the loss of traffic capacity varies linearly with the percentage of local traffic. In particular, when all traffic is local, the traffic capacity of the hub-based architecture is reduced by a factor of 2 when compared to the opaque and slotted WDM architectures.

Despite its lower transmission and reception capacity, the time-slotted WDM architecture is able to reach a traffic capacity nearly equivalent to that of opaque networks in all the considered traffic scenarios. By allowing the capacity of each wavelength channel to be shared among multiple sourcedestination pairs, the slotted WDM architecture exploits the network capacity as efficiently as an opaque solution, while minimizing the number of required receivers and transmitters.

Still considering the above case study, we apply the models presented in Sec.IV-B to determine the maximum offered 


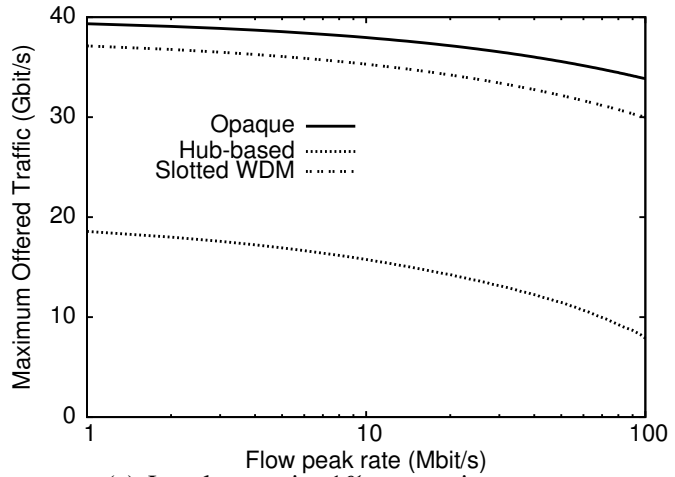

(a) Local scenario, $1 \%$ congestion rate.

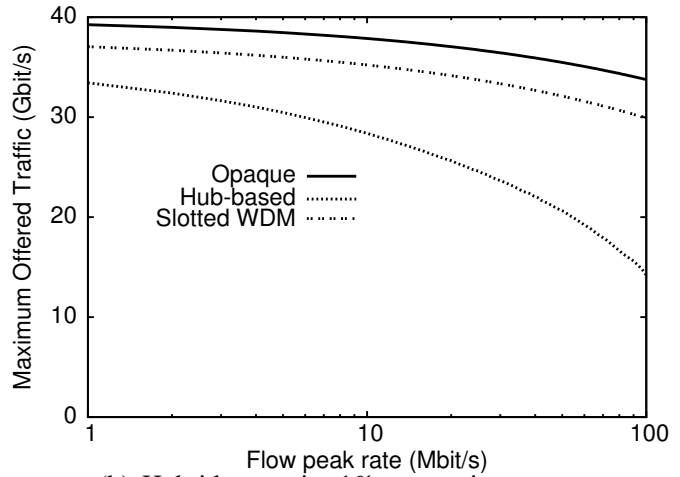

(b) Hybrid scenario, $1 \%$ congestion rate.

Fig. 7. Maximum offered traffic for a congestion rate of $1 \%$ under the Local and Hybrid scenarios.

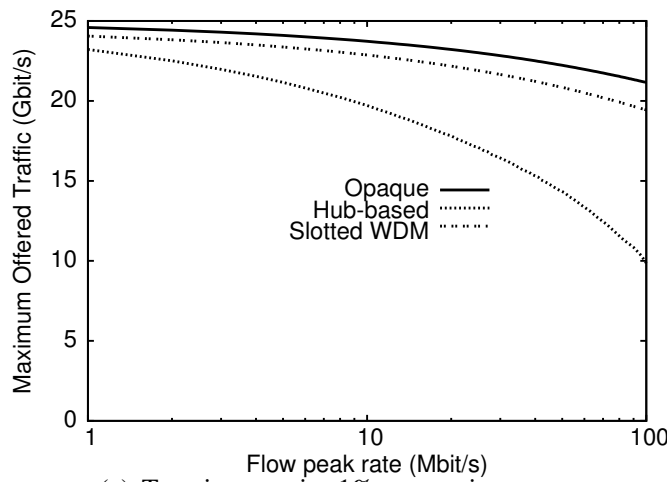

(a) Transit scenario, $1 \%$ congestion rate.

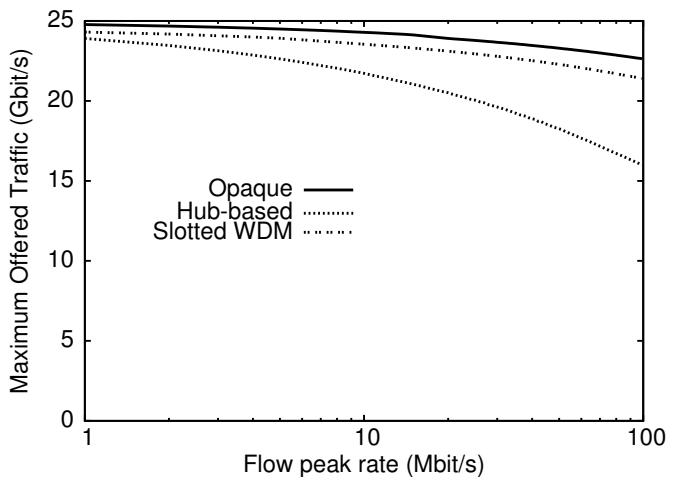

(b) Transit scenario, $10 \%$ congestion rate.

Fig. 8. Maximum offered traffic for congestion rates of $1 \%$ and $10 \%$ under the Transit traffic scenario.

traffic. Figg.7(a), 7(b) and 8(a) depict the maximum offered traffic for different flow peak rates and for a target congestion rate of $1 \%$ in the Local, Transit and Hybrid traffic scenarios. In the opaque architectures, the $W_{1}=2$ wavelengths of each network link are equivalent: data can be transmitted to a specific destination using any of the two wavelengths. This translates to a total link capacity of $W_{1} R_{1}=20 \mathrm{Gbit} / \mathrm{s}$. In the slotted WDM architecture, network nodes receive data on a specific, predefined wavelength channel which means that the shared resource in this architecture is the wavelength channel of rate $R_{1}=10 \mathrm{Gbit} / \mathrm{s}$. Finally, in the hub-based architecture, network nodes communicate by means of $R_{2}=1 \mathrm{Gbit} / \mathrm{s}$ wavelength channels. Consequently, the probability that flows are constrained by the link capacity is considerably higher in the hub-based architecture, leading to lower maximum sustainable traffic. Note that the total amount of traffic that can be sustained by the opaque and the slotted WDM architecture is sensibly close. We further notice that due to its lack of sharing, the hub-based architecture performs very poorly, especially when the traffic is distributed among the different access nodes, as in the Local scenario.

Fig.8(b) depicts the maximum offered traffic as a function of the flow peak rate for a congestion rate of $10 \%$ in the Transit traffic scenario. Allowing a higher congestion rate yields higher maximum sustainable traffic. However, the max- imum traffic sustained by the hub-based architecture is still considerably lower than the maximum traffic of the opaque and slotted WDM architectures, indicating a poor utilization of the available resources.

\section{Cost and power consumption}

In the case of the opaque and the hub-based architectures, the components required to build the network nodes are currently available on the market. Thus, we selected the appropriate components from the ones provided by network equipment manufacturers [18], [19]. To evaluate the cost of these architectures we relied on market data [20]. However, the fast tunable lasers and the $10 \mathrm{Gbit} / \mathrm{s}$ burst-mode receivers required to build an OPADM are still in the development phase and are expected to become available only in the near future. To accurately predict the cost and power consumption of such components, we rely both on data of the most sophisticated currently available components and on the estimations reported in [21]. All costs are normalized to the cost of a $10 \mathrm{Gbit} / \mathrm{s}$ transceiver having a transmission range of $40 \mathrm{~km}$. To assess the power consumption, we collected data about each component from data sheets provided by network equipment manufacturers [18], [19].

Tab.II-V summarize the normalized cost and power consumption for the components of SONET/SDH nodes, Ethernet 
Carrier Grade switches, OADMs and OPADMs, respectively. We note that in the case of the hub-based architecture, the cost of the hub node is nearly 8 times higher than that of an access node. The discrepancy would become even higher for a larger number of access nodes $N$, questioning the scalability of such architecture.

\begin{tabular}{|l|c|c|}
\hline Component & Cost & Power consumption \\
\hline \hline Base Node Access & 0.5 & $216 \mathrm{~W}$ \\
\hline Base Node Hub & 0.5 & $294 \mathrm{~W}$ \\
\hline 1 port 10 Gbit/s slot cards & 4.0 & $40 \mathrm{~W}$ \\
\hline 8 port downstream Slot cards & 1.4 & $50 \mathrm{~W}$ \\
\hline 10 Gbit/s OC-192/STM-64 XFP & 0.4 & $3 \mathrm{~W}$ \\
\hline
\end{tabular}

TABLE II

NORMALIZED COMPONENT COSTS AN POWER CONSUMPTION FOR SONET/SDH NODES.

\begin{tabular}{|l|c|c|}
\hline Component & Cost & Power consumption \\
\hline \hline Access Node & 3.5 & $300 \mathrm{~W}$ \\
\hline Base Node Hub & 4.0 & $280 \mathrm{~W}$ \\
\hline 4 port 10 Gbit/s slot cards & 3.0 & $60 \mathrm{~W}$ \\
\hline 10 Gbit/s X2 Transponder & 1.0 & $3 \mathrm{~W}$ \\
\hline
\end{tabular}

TABLE III

NORMALIZED COMPONENT COSTS AN POWER CONSUMPTION FOR ETHERNET CARRIER GRADE NODES

In Sec.V we showed that the total traffic that can be sustained by each architecture depends on the considered traffic scenario. In Fig.9, we compare the cost and power consumption per carried Gbit/s for each studied architecture and each traffic scenario. Due to its complex control and inherent redundancies between layers 1, 2 and 3, the SONET/SDH architecture presents the highest cost and power consumption per Gbit/s. We note that, despite requiring only low cost $1 \mathrm{Gbit} / \mathrm{s}$ components, the hub-based solution does not yield the lowest cost per Gbit/s in all traffic scenarios. Indeed,

\begin{tabular}{|l|c|c|}
\hline Component & Cost & Power consumption \\
\hline \hline Base Node Access & 1.4 & $110 \mathrm{~W}$ \\
\hline Base Node Hub & 4.2 & $280 \mathrm{~W}$ \\
\hline Slot card 1Gbit/s & 4.0 & $60 \mathrm{~W}$ \\
\hline 1 Gbit/s Transceiver & 0.4 & $1 \mathrm{~W}$ \\
\hline 10 channel MUX/DMUX & 0.3 & $1.5 \mathrm{~W}$ \\
\hline
\end{tabular}

TABLE IV

NORMALIZED COMPONENT COSTS AND POWER CONSUMPTION FOR AN OADM.

\begin{tabular}{|l|c|c|}
\hline Component & Cost & Power consumption \\
\hline \hline Base Node Access & 1.40 & $110 \mathrm{~W}$ \\
\hline Base Node Hub & 3.50 & $300 \mathrm{~W}$ \\
\hline 2 channel MUX/DMUX & 0.10 & $1.5 \mathrm{~W}$ \\
\hline Semiconductor Optical Amplifier & 0.80 & $0.2 \mathrm{~W}$ \\
\hline 10 Gbit/s Transceiver (TT, BMR) & 1.50 & $3 \mathrm{~W}$ \\
\hline $1 \times 2 \mathrm{Passive}$ splitter & 0.01 & - \\
\hline $155 \mathrm{Mb} / \mathrm{s}$ Transceiver $(\mathrm{CC})$ & 0.10 & $1 \mathrm{~W}$ \\
\hline
\end{tabular}

TABLE V

NORMALIZED COMPONENT COSTS AND POWER CONSUMPTION FOR OPADM. due to its poor traffic capacity, the hub-based architecture turns out to be more costly and power consuming than the slotted WDM solution in a Local traffic scenario. We also observe that the two transparent solutions are expected to reduce power consumption by up to $75 \%$ with respect to today's SONET/SDH networks. By reducing the amount of required equipment (i.e., number of transmitters and receivers), the slotted WDM architecture outperforms an Ethernet-based architecture both in terms of cost and power consumption.

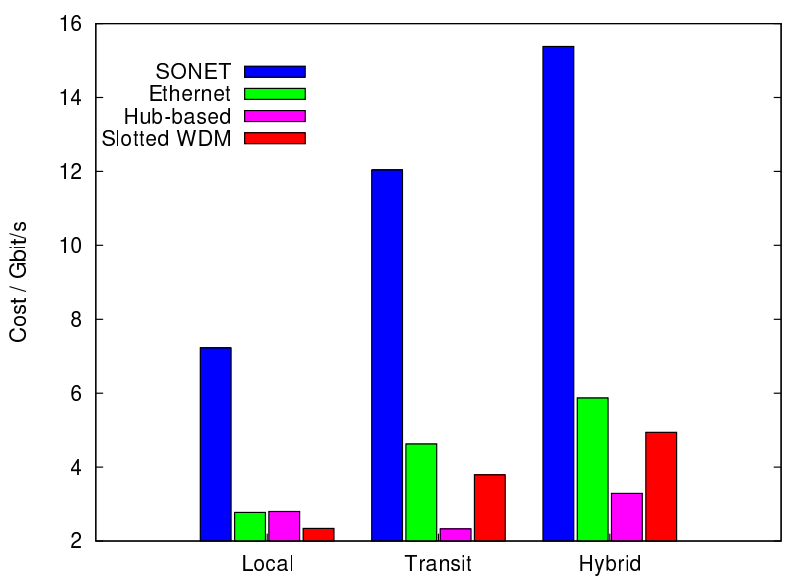

(a) Normalized cost per Gbit/s

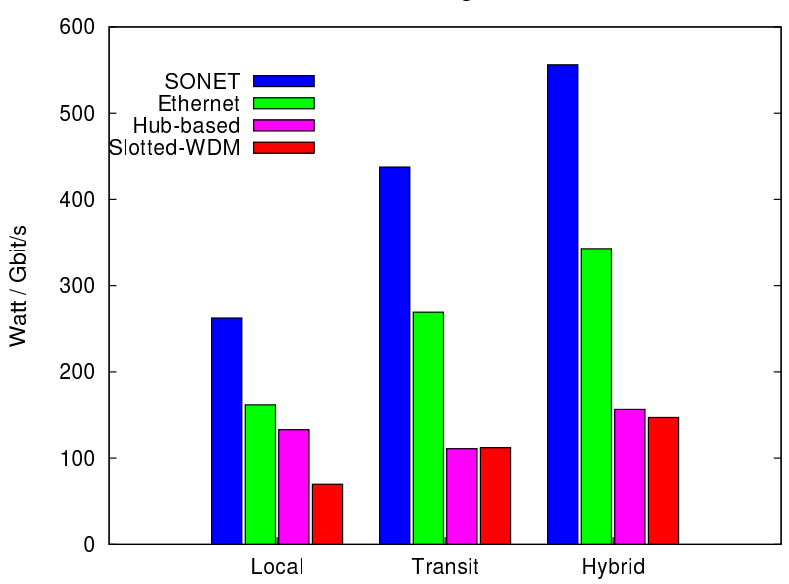

(b) Power consumption per Gbit/s

Fig. 9. Normalized cost and power consumption per Gbit/s for each architecture in the Local, Transit and Hybrid scenario.

\section{E. Impact of network parameters}

Our case study considered the specific case of a metro network having $N=20$ nodes that share a given number of wavelength channels. This allowed us to evaluate the cost and power consumption based on commercially available network equipment. We now wish to assess the impact of these two parameters on the overall network cost. For simplicity, we focus here only on transceiver cost disregarding the fixed cost of nodes. Fig.10 represents the impact of $N$ and $W$ on the network cost. As expected, increasing the number of nodes $N$ causes an almost linear increase of network cost for 
all architectures. On the other hand, increasing the network capacity by increasing the number of wavelengths $W$ would obviously have a greater impact on the cost of the opaque architecture in which every node needs to be equipped with $W$ fixed transceivers. The network power consumption is clearly expected to follow the same trend.

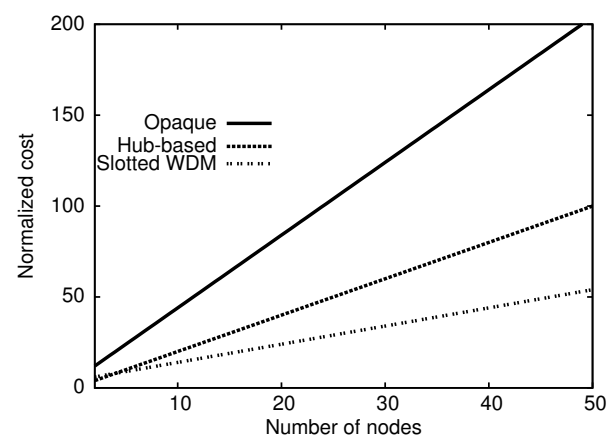

(a) Impact of the number of nodes $N$ when $W=4$

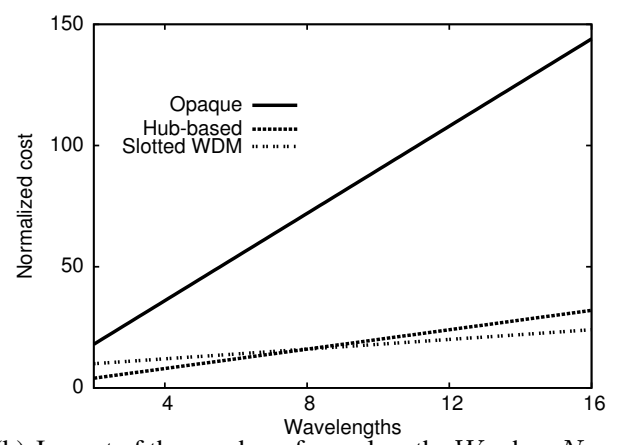

(b) Impact of the number of wavelengths $W$ when $N=$ 8

Fig. 10. Normalized network cost due to transceivers as a function of $N$ and $W$.

\section{CONCLUSION}

We provided models for evaluating the cost, power consumption and traffic capacity of different network architectures. Then, we applied these models to compare four architectures suitable for a metropolitan area network. Our results indicate that allowing transit traffic to bypass intermediate nodes in the optical domain would reduce power consumption by up to $75 \%$ with respect to today's opaque SONET/SDH networks. We studied two different architectures which enable optical bypass of transit traffic. We showed that a time-slotted WDM architecture in which the capacity of each wavelength is dynamically shared among several source-destination pairs is able to attain a level of performance equivalent to that of opaque networks while minimizing the number of required transmitters and receivers and thus the total network cost.
As future work we intend to extend the present study by comparing the cost and power consumption of the considered architectures in a dynamic traffic environment, when interfaces are switched off during periods of low network load.

\section{REFERENCES}

[1] "The business case for optical ethernet vs. legacy SONET/SDH in a metro service provider data network," Metro Ethernet Network, Tech. Rep., 2003.

[2] "Ethernet in the MAN: New technology creates new revenue opportunities," Sorento Networks, Tech. Rep., 2003.

[3] H. Schmidtke and A. Gibbemeyer, "Five reasons to adopt layer 2 Ethernet switching over DWDM networks now," Tech. Rep., 2006.

[4] M. Maier, Optical Switching Networks. Cambridge University Press, 2008.

[5] N. Le Sauze, A. Dupas, E. Dotaro, L. Ciavaglia, M. Nizam, A. Ge, and L. Dembeck, "A novel, low cost optical packet metropolitan ring architecture," in 27th European Conference and Exhibition on Optical Communication (ECOC), Sep. 2001.

[6] I. White, M. Rogge, K. Shrikhande, and L. Kazovsky, "A summary of the HORNET project: a next-generation metropolitan area network," Selected Areas in Communications, IEEE Journal on, vol. 21, no. 9, pp. 1478 - 1494, Nov. 2003.

[7] C. Jelger and J. Elmirghani, "A slotted MAC protocol for efficient bandwidth utilization in WDM metropolitan access ring networks," Selected Areas in Communications, IEEE Journal on, vol. 21, no. 8, pp. 1295 - 1305, Oct. 2003.

[8] A. Bianco, J. Finochietto, G. Giarratana, F. Neri, and C. Piglione, "Measurement-based reconfiguration in optical ring metro networks," Lightwave Technology, OSA Journal of, vol. 23, no. 10, pp. 3156 3166, Oct. 2005.

[9] M. Yuang, I.-F. Chao, and B. Lo, "HOPSMAN: An experimental optical packet-switched metro WDM ring network with high-performance medium access control," Optical Communications and Networking, IEEE/OSA Journal of, vol. 2, no. 2, pp. 91-101, Feb. 2010.

[10] Intune networks. [Online]. Available: http://www.intunenetworks.com/

[11] D. Chiaroni et al., "Demonstration of the interconnection of two optical packet rings with a hybrid optoelectronic packet router," in 36th European Conference and Exhibition on Optical Communication (ECOC), Sep. 2010

[12] T. Bonald, R.-M. Indre, S. Oueslati, and C. Rolland, "Throughput delay trade-offs in slotted WDM ring networks," in BROADNETS, 2010.

[13] D. Chiaroni et al., "Packet OADMs for the next generation of ring networks," Bell Lab. Tech. J., vol. 14, no. 4, pp. 265-283, Feb. 2010.

[14] B. Mukherjee, Optical WDM Networks. Springer-Verlag New York, Inc., 2006.

[15] "Business case for carrier Ethernet services," CISCO Systems, Tech. Rep., 2005.

[16] N. Project, "Deliverable d2.4: Migration guidelines with economic assessment and new business opportunities generated by NOBEL phase 2," Mar 2008.

[17] R. Huelsermann, M. Gunkel, C. Meusburger, and D. Schupke, "Cost modeling and evaluation of capital expenditures in optical multilayer networks," J. Opt. Netw., vol. 7, no. 9, pp. 814-833, Sep. 2008.

[18] "Cisco data sheets, available: http://www.cisco.com."

[19] "Data sheets taken from www.oequest.com."

[20] "Data taken from www.router-switch.com."

[21] K. Grobe, M. Roppelt, A. Autenrieth, and M. Eiselt, "Cost and energy consumption analysis of advanced WDM-PONs," IEEE Communications Magazine, 2011. 